\title{
Bilateral Postsynaptic Actions of Pyramidal Tract and Reticulospinal Neurons on Feline Erector Spinae Motoneurons
}

\author{
Mary Pauline Galea, ${ }^{1,2}$ Ingela Hammar, ${ }^{1}$ Elin Nilsson, ${ }^{1}$ and Elzbieta Jankowska ${ }^{1}$ \\ ${ }^{1}$ Department of Physiology, Sahlgrenska Academy, University of Gothenburg, 40530 Gothenburg, Sweden, and ${ }^{2}$ School of Physiotherapy, The University of \\ Melbourne, Parkville, Victoria 3010, Australia
}

Trunk muscles are important for postural adjustments associated with voluntary movements but little has been done to analyze mechanisms of supraspinal control of these muscles at a cellular level. The present study therefore aimed to investigate the input from pyramidal tract (PT) neurons to motoneurons of the musculus longissimus lumborum of the erector spinae and to analyze to what extent it is relayed by reticulospinal (RS) neurons. Intracellular records from motoneurons were used to evaluate effects of electrical stimulation of medullary pyramids and of axons of RS neurons descending in the medial longitudinal fasciculus (MLF). The results revealed that similar synaptic actions were evoked from the ipsilateral and contralateral PTs, including disynaptic and trisynaptic EPSPs and trisynaptic IPSPs. Stimulation of the MLF-evoked monosynaptic and disynaptic EPSPs and disynaptic or trisynaptic IPSPs in the same motoneurons. All short-latency PSPs of PT origin were abolished by transection of the MLF, while they remained after transection of PT fibers at a spinal level. Hence, RS neurons might serve as the main relay neurons of the most direct PT actions on musculus (m.) longissimus. However, longer-latency IPSPs remaining after MLF or PT spinal lesions and after ipsilateral or contralateral hemisection of spinal cord indicate that PT actions are also mediated by ipsilaterally and/or contralaterally located spinal interneurons. The bilateral effects of PT stimulation thereby provide an explanation why trunk movements after unilateral injuries of PT neurons (e.g., stroke) are impaired to a lesser degree than movements of the extremities.

\section{Introduction}

Despite a large number of studies on postural adjustments, the neuronal networks underlying these adjustments remain to a great extent unknown. This is true in particular for supraspinal control of postural adjustments of back muscles, as most previous studies were concerned with adjustments of the whole body, or of proximal limb muscles rather than trunk muscles.

The importance of back muscles for stabilizing the trunk and enabling effective actions of the extremities is beyond doubt. Deficits in trunk control and postural asymmetry occur after stroke-related unilateral damage to pyramidal tract (PT) neurons (Davies, 1990; van Nes et al., 2008) as well as after more selective lesions of medullary pyramids in animals (Lawrence and Kuypers, 1968). Deficits in postural control are also seen in patients with idiopathic low back pain (Zedka et al., 1999; Hodges et al., 2009). Studies in poststroke hemiplegic patients have shown that the erector spinae muscles are particularly important for stabilizing the trunk and its movements, and

\footnotetext{
Received Sept. 30, 2009; revised Nov. 20, 2009; accepted Nov. 30, 2009.

The study was supported by grants from National Institute of Neurological Disorders and Stroke/National Institutes of Health (R01 NS040863) and the Swedish Research Council (15393-01A to E.J. and 522-2005-7255 to I.H.).

Correspondence should be addressed to Elzbieta Jankowska, Department of Physiology, Sahlgrenska Academy, University of Gothenburg, Medicinaregatan 11,Box 432,40530 Gothenburg, Sweden. E-mail: Elzbieta.Jankowska@ physiol.gu.se.

DOI:10.1523/JNEUROSCI.4859-09.2010

Copyright $\odot 2010$ the authors $\quad 0270-6474 / 10 / 300858-12 \$ 15.00 / 0$
}

that, in contrast to the abdominal muscles, their activity is relatively unimpaired (Dickstein et al., 1999). Residual function of the erector spinae muscles after unilateral stroke might indicate a stronger subcortical than cortical control of these muscles, or stronger ipsilateral actions of PT neurons in the noninjured hemisphere (for references, see Dum and Strick, 1996; Jankowska and Edgley, 2006).

Both corticospinal and reticulospinal neurons have been shown to be involved in postural adjustments (Massion, 1992, 1994; for recent references see Deliagina et al., 2008; Schepens et al., 2008). However, activity of these neurons has primarily been related to limb movements or to anticipatory or corrective postural adjustments of the whole body (Alstermark et al., 1985; Luccarini et al., 1990; Chabran et al., 2001; Schepens and Drew, 2004, 2006; Deliagina et al., 2006, 2008; Caronni and Cavallari, 2009a,b) and there are only scarce data on their actions on back muscles. For example, stimuli applied in the reticular formation were found to evoke short-latency EMG responses in epiaxial muscles, including back muscles in the rat (Cottingham et al., 1988) and to facilitate likely monosynaptic reflexes from these muscles (Brink et al., 1981). The relative contribution of PT and reticulospinal neurons to postsynaptic excitation and inhibition of neck motoneurons (Wilson and Yoshida, 1968; Alstermark et al., 1985) and of reticulospinal neurons on back motoneurons in thoracic segments (Peterson et al., 1979) was examined in the cat, but no reports have been made on actions of these neurons on lumbar back motoneurons. 


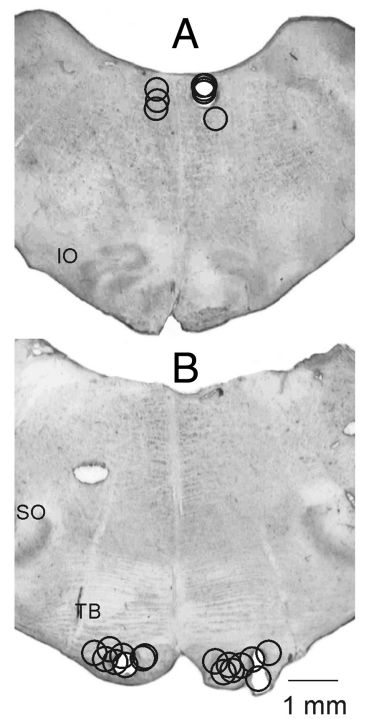

C LPT $100 \mu \mathrm{A}$ LPT $100 \mu \mathrm{A}$ RPT $100 \mu \mathrm{A}$ RPT $100 \mu \mathrm{A}$

LPT $150 \mu \mathrm{A}$ R PT $150 \mu \mathrm{A}$

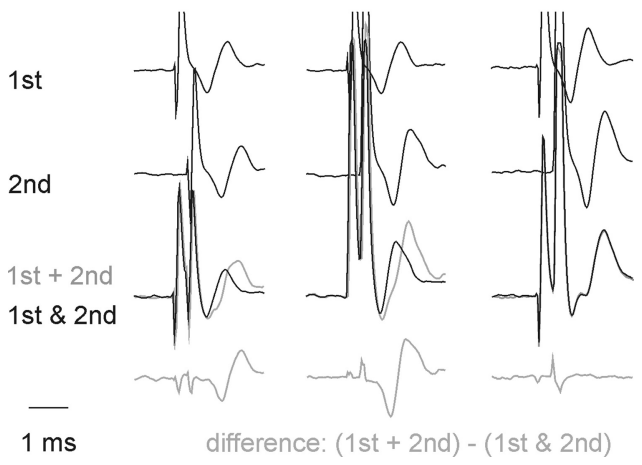

Figure 1. Stimulation sites and collision tests. $\boldsymbol{A}$, Stimulation sites within the MLF and at its lateral borders as indicated by electrolytic lesions made at the end of the experiments. Stimulation sites to the left and right of the midline are for preparations in which the spinal cord was intact, or was hemisected on the left side, respectively,. I0, inferior olive. $\boldsymbol{B}$, Stimulation sites within the left and right PTs. SO, superior olive; TB, trapezoid body. $C, D$, Examples of collision tests of effects of stimuli applied within the left or right PT, respectively. They show that the second stimulus applied $\sim 0.3 \mathrm{~ms}$ after the first stimulus fell within the refractory period and failed to activate the same fibers. This is indicated by a difference between effects of the first and second stimuli applied together within this interval (black trace) and the sum of their effect when applied one at a time (gray trace) shown at the bottom. $\boldsymbol{E}$, Examples of records indicating that the stimuli applied within the left and right PT did not stimulate the same fibers because effects of the two stimuli were identical when applied jointly and when applied separately; note that that bottom trace did not show differences between them. In the illustrated case no spread of current was found from one PT to another at $150 \mu \mathrm{A}$ but in other experiments this was the case only for stimuli $\leq 110$ or $100 \mu \mathrm{A}$.

The main aim of the reported experiments was therefore to investigate the effects of pyramidal tract (PT) fibers on intracellularly recorded motoneurons innervating the $\mathrm{m}$. longissimus lumborum, the most medial component of the erector spinae muscle group, and to compare effects of contralateral and ipsilateral PT neurons. We further aimed to investigate whether the corticospinal actions on the erector spinae motoneurons are relayed by reticulospinal neurons as found for PT actions on hindlimb motoneurons (Stecina and Jankowska, 2007).

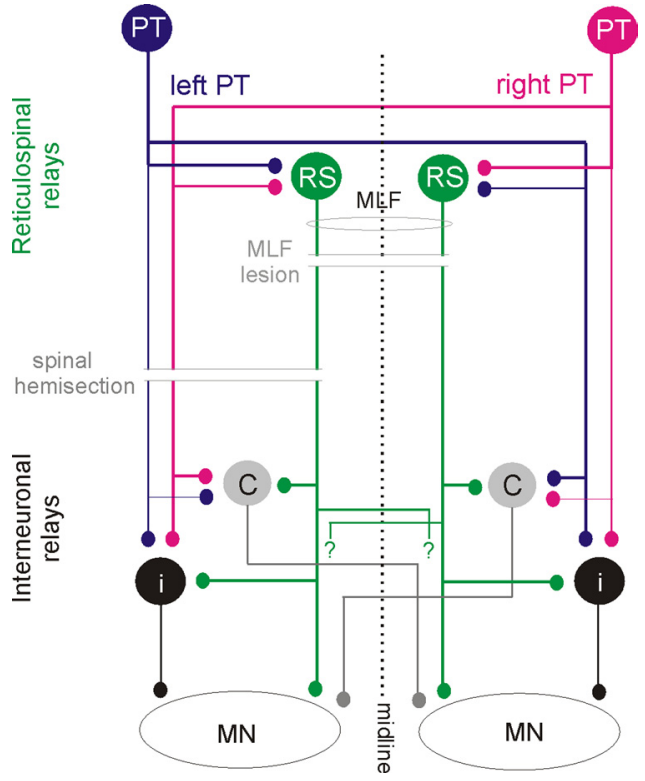

Figure 2. Diagram of hypothesized neuronal pathways from PT and RS neurons to back motoneurons and of two experimental approaches used to verify them. Blue and red circles and lines represent left and right side PT neurons and their projections. Green circles represent RS neurons with axons descending within the MLF; crossed segmental axon collaterals with unspecified target cells are indicated with "?". Gray and black circles represent spinal target neurons of the PT and RS neurons, black projecting ipsilaterally and gray contralaterally. Thinner lines denoting ipsilaterally descending PT fibers and axons of interneurons indicate weaker projections. Parallel horizontal lines indicate lesions of the MLF (made to test the relative contribution of RS neurons) and the spinal cord hemisection (made to test the relative contribution of all descending tract and propriospinal tract neurons on the left side as well as the relative contribution of ipsilaterally and contralaterally projecting spinal interneurons).

\section{Materials and Methods}

Preparation. The experiments were performed on 8 deeply anesthetized adult cats weighing $2.5-4.5 \mathrm{~kg}$. All experimental procedures were approved by the University of Gothenburg Ethics Committee and followed National Institutes of Health and European Union guidelines for animal care. Anesthesia was induced with sodium pentobarbital $(40-44 \mathrm{mg} / \mathrm{kg}$, i.p.) and maintained with intermittent doses of $\alpha$-chloralose (RhônePoulenc Santé, France; doses of $5 \mathrm{mg} / \mathrm{kg}$ administered every $1-3 \mathrm{~h}$, up to $50 \mathrm{mg} / \mathrm{kg}$, i.v.). During recordings, neuromuscular transmission was blocked by pancuronium bromide (Pavulon, Organon; $\sim 0.2 \mathrm{mg} / \mathrm{kg} / \mathrm{h}$, i.v.) and the animals were artificially ventilated. Additional doses of $\alpha$-chloralose were given when increases in blood pressure or heart rate, both of which were continuously monitored, were evoked by stimulation, or if the pupils dilated. Mean blood pressure was kept at 90-130 $\mathrm{mmHg}$ (with the exception of 2 cats in which it dropped to $80-90 \mathrm{mmHg}$ toward the end of the experiments) and end-tidal concentration of $\mathrm{CO}_{2}$ at $\sim 4 \%$ by adjusting the parameters of artificial ventilation and the rate of a continuous infusion of a bicarbonate buffer solution with $5 \%$ glucose (1-2 $\mathrm{ml} / \mathrm{h} / \mathrm{kg}$ ). The core body temperature was kept at $\sim 37.5^{\circ} \mathrm{C}$ by servocontrolled infrared lamps. The experiments were terminated by a lethal dose of anesthetic followed by formalin perfusion resulting in cardiac arrest.

A laminectomy exposed the first to fourth lumbar (L1-L4) and low thoracic (Th10-Th11) segments and a small area at the border between the third and fourth cervical (C3-C4) segments of the spinal cord. The intermediate branches of the dorsal rami innervating $\mathrm{m}$. longissimus lumborum were exposed at L2 and L3 levels bilaterally. The approach was made through a longitudinal split between the multifidus and longissimus muscles. The nerves were identified close to where they emerged in the intertransverse space and dissected from the surrounding tissue. They were ligated, transected and mounted on two pairs of stimulating electrodes in a paraffin oil pool.

The caudal part of the cerebellum was exposed by a craniotomy and tungsten electrodes (impedance $30-150 \mathrm{k} \Omega$ ) were placed in the left 
and/or right pyramids (PT) in the caudal part of the medulla and in the left or right medial longitudinal fasciculus (MLF). The electrodes were inserted at an angle of 35 degrees (with the tip directed rostrally). The initial targets were at Horsley-Clarke coordinates P5, L and R 1.4, $\mathrm{H}-10$ for the left and right PT and P10, L or R $0.5, \mathrm{H}-5$ for the MLF, respectively. However, the final positions of the electrodes were adjusted on the basis of records of descending volleys from the surface of the lateral funiculus at the Th10 and the C3-C4 segments. The electrodes were left at sites from which distinct descending volleys were evoked at stimulus intensities of 10-20 $\mu \mathrm{A}$ or less. At the end of the experiments these sites were marked with electrolytic lesions and their location subsequently verified on $100 \mu \mathrm{m}$ thick frontal sections of the brainstem cut in the plane of insertion of the electrodes using a freezing microtome. The MLF electrodes were usually placed at the level corresponding to the rostral border of the inferior olive and the PT electrodes at the level of the trapezoid body. In Figure 1, $A$ and $B$, the electrolytic lesions indicating stimulation sites in all 8 experiments are projected onto sections from one of these experiments.

In 2 experiments the MLF fibers were transected by a $3 \mathrm{~mm}$ wide blade mounted on a Horsley-Clarke manipulator and introduced in the midline a few $\mathrm{mm}$ rostral to the obex and a few $\mathrm{mm}$ caudal to the electrodes located in the MLF and the PTs. The blade was advanced until descending volleys evoked by $100 \mu \mathrm{A}$ stimuli applied in the MLF disappeared (in one experiment) or were reduced to $\sim 90 \%$ (in the other experiment). Descending volleys from the PTs recorded at the C3 level were either unchanged or only moderately decreased (see Results).

PT fibers descending to the spinal cord were transected by a lesion of the dorsal part of the lateral funiculus in the $\mathrm{C} 3$ segment (in one experiment), by lesions of the whole lateral funiculus in the Th11 segment (in 2 experiments) and by hemisection of the spinal cord in the Th11 segment (in 2 experiments). The C3 lesion was made under control of recording of descending volleys from the two PTs and from the MLF at the level of the C4 segment. The lesion was extended until the PT volleys disappeared while the MLF volleys remained unchanged (see Results). The lesions of the lateral funiculus covered the whole area of the ventral part of this funiculus within which propriospinal neurons located in the C3-C4 segments descend (Illert et al., 1978; Lundberg, 1979) but encroached on the lateral part of the ventral funiculus.

Stimulation and recording. Nerves innervating $\mathrm{m}$. longissimus lumborum were stimulated with constant voltage stimuli $(0.2 \mathrm{~ms}$ duration, at intensities $2-5$ times threshold intensities for the most sensitive fibers in the nerve). For activation of fibers of the reticulospinal and corticospinal tracts, constant current cathodal stimuli $(0.2 \mathrm{~ms}, 25-100 \mu \mathrm{A})$ were applied. The stimuli were applied as single stimuli (at $\sim 3 \mathrm{~Hz}$ ) or in trains of 2-6 stimuli at 300 or $400 \mathrm{~Hz}$ (delivered at $\sim 3 \mathrm{~Hz}$ ). Near maximal stimuli applied in the MLF were expected to activate a large proportion of pontoand medullary reticulospinal tract fibers (Jankowska et al., 2003), as needed for purposes of this study, but also other descending fibers which might complicate the interpretation of the results. However, any coactivated tectospinal and vestibulospinal tract fibers arising from the medial vestibular nucleus would not project as far caudally as the lumbar segevoked by the last stimuli.

\section{Contralateral PT}

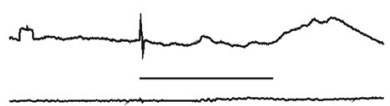

$0.2 \mathrm{mV}$

$(\mathrm{A}-\mathrm{L})$

$4 \mathrm{~ms}$

$(\mathrm{A}-\mathrm{L})$
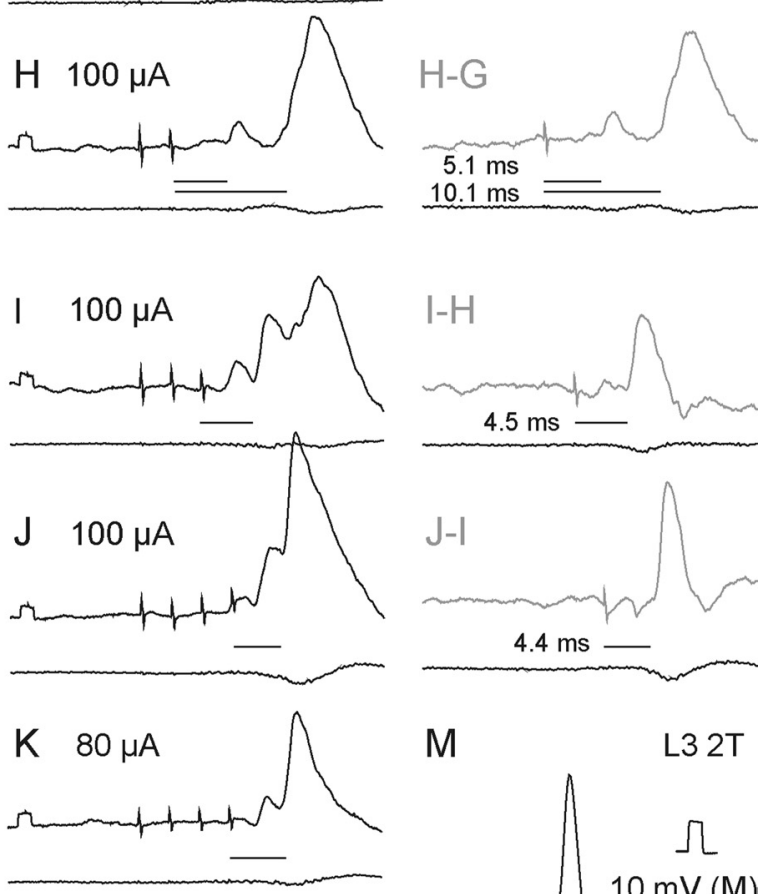

M

L3 2T

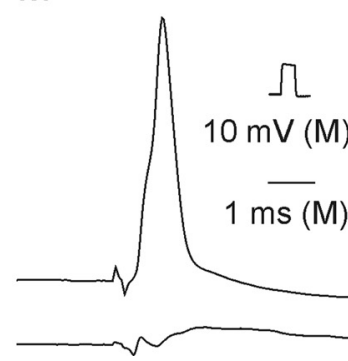

Figure 3. Examples of EPSPs evoked from the ipsilateral and contralateral PTs. Upper traces in $\boldsymbol{A}-\boldsymbol{M}$ are intracellular records from a motoneuron in a preparation with the spinal cord intact. Lower traces are simultaneously obtained cord dorsum porentials. Averages of 20 records. $A-D$ and $G-I$, EPSPs evoked by increasing numbers of stimuli at the same pulses at the beginning of the intracellular records are calibration pulses. Horizontal lines indicate latencies of potentials

ments and fibers from the lateral vestibular (Deiter's) nucleus would not be activated by stimuli of $100 \mu \mathrm{A}$ (Nyberg-Hansen and Mascitti, 1964; Hongo et al., 1975). Hence effects evoked in the lumbar segments following MLF stimulation can be attributed to reticulospinal fibers.

Descending volleys were recorded from the surface of the dorsal columns at the C3-C4 segments for PT volleys, or from the surface of the left or right lateral funiculus at the Th10 and L2 or L3 segments, for MLF volleys. The volleys were recorded monopolarly via intact dura mater.

Glass micropipettes filled with $2 \mathrm{~m}$ solution of potassium citrate were used for intracellular recordings from motoneurons; the tips of the electrodes were broken to $\sim 1.5 \mu \mathrm{m}$ with an impedance of 3-5 $\mathrm{M} \Omega$. Motoneurons innervating $\mathrm{m}$. longissimus were identified by antidromic activation following stimulation of one of the dissected nerves.

Sample. The reported results are based on the analysis of records from 141 motoneurons in the L2 and L3 segments. Of these, 120 were identified as innervating $\mathrm{m}$. longissimus lumborum by antidromic activation following stimulation of muscle nerve branches of the L2 or L3 spinal nerves and 21 motoneurons by monosynaptic input from these nerve branches. These motoneurons were recorded in preparations in which 
With intact spinal cord

A

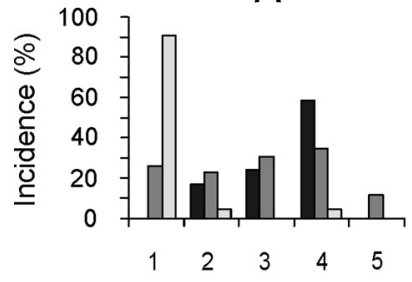

B

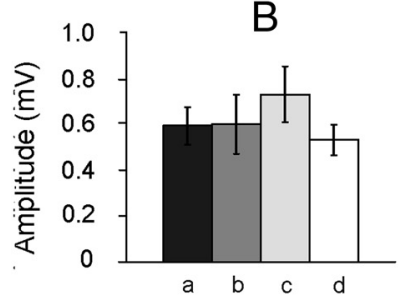

After DLF lesion

C

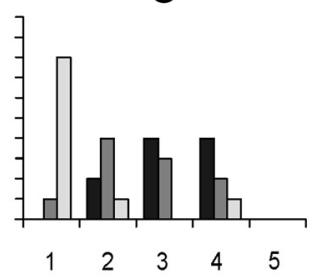

After LF lesion

$\mathrm{E}$

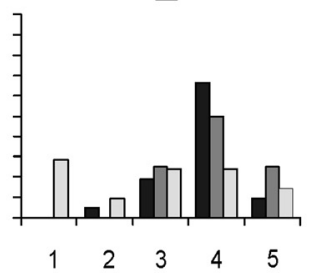

After ipsi hemisection

G

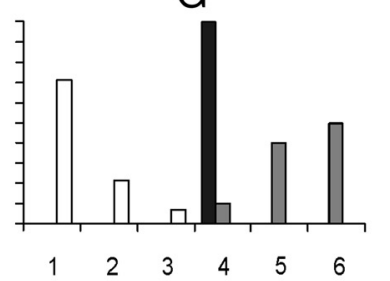

After co hemisection

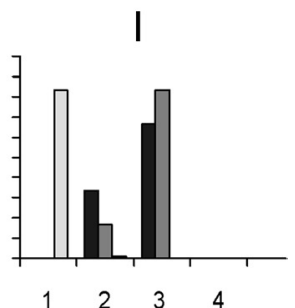

After 1 st, 2 nd, 3rd, 4th, 5th and 6th stimulus in a train

D $\quad F$

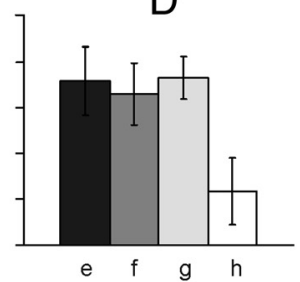

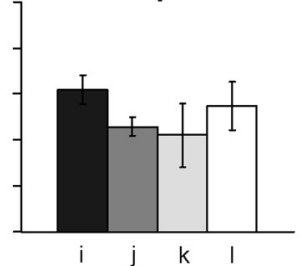
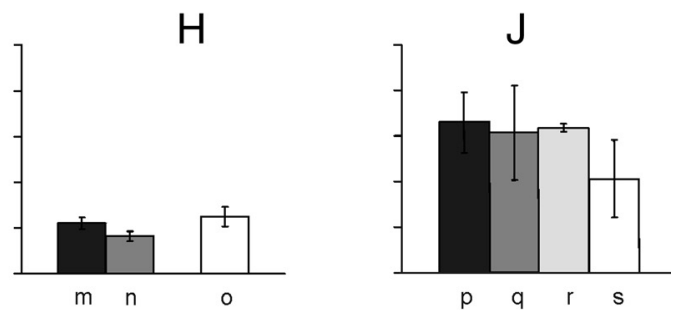

PSPs evoked from the contralateral PT ( $\square$ ), ipsilateral PT ( $\square$ ) and ipsilateral MLF monosynaptic ( $\square$ ) and disynaptic ( $\square$ )

Figure 4. Comparison of stimulus related incidence and amplitudes of short-latency EPSPs evoked from the contralateral and ipsilateral PTs and from the MLF. $\boldsymbol{A}$, Proportions of motoneurons recorded in preparations with the spinal cord intact in which the first EPSPs were evoked by the first-fifth stimuli (100 $\mu$ A). The plots are for EPSPs with latencies not exceeding $5 \mathrm{~ms}$ for the contralateral PT $(n=29)$ and ipsilateral PT $(n=26)$, and for monosynaptic EPSPs from the MLF $(n=22)$. In the remaining motoneurons no EPSPs were induced by such stimuli or no comparison was made of effects of the varying numbers of stimuli. $C, E, G, I$, as in $\boldsymbol{A}$ but for preparations in which descending tract fibers were transected, as indicated, and for a mixture of monosynaptic and disynaptic rather than only monosynaptic EPSPs from the MLF in $\boldsymbol{G}$. The lesions were of the dorsal part of the lateral funiculus (DLF; $n=12$ ), the whole lateral funiculus (LF; $n=22)$, ipsilateral hemisection of the spinal cord $(n=14)$, and contralateral hemisection $(n=12) . \boldsymbol{B}, \boldsymbol{D}, \boldsymbol{F}, \boldsymbol{H}, \boldsymbol{J}$, Amplitudes of EPSPs evoked in the same samples of motoneurons. Amplitudes of EPSPs with characteristics of disynaptic EPSPs from the PTs were measured for those evoked by the second effective stimuli after having subtracted PSPS evoked by previous stimuli but without including EPSPs preceded by IPSPs evoked by earlier stimuli, or cut short by IPSPS that followed them. Amplitudes of monosynaptic EPSPs from the MLF were measured for those evoked by single stimuli in $\boldsymbol{B}, \boldsymbol{D}, \boldsymbol{F}$, and $\boldsymbol{J}$ but for combined monosynaptically and disynaptically evoked EPSPs in $\boldsymbol{H o}$ because of difficulties in differentiating between them. Amplitude of disynaptic EPSPs from the MLF were measured only in motoneurons in which no monosynaptically evoked EPSPs were evoked. No statistically significant differences were found between amplitudes of EPSPs evoked from the two PTs in the same preparation (except in $F i, j^{*}$ ), or between those from PTs and monosynaptic EPSPs from the MLF in the same preparation. In contrast highly statistically significant differences were found between EPSPs evoked after ipsilateral hemisection and in other preparations $\left(\boldsymbol{a}-\boldsymbol{m}^{* *}, \boldsymbol{b}-\boldsymbol{n}^{*}, \boldsymbol{d}-\boldsymbol{0}^{* *} ;{ }^{*} p<0.05,{ }^{* *} p<0.01\right)$.

Table 1. Latencies and incidence of short-latency EPSPs and IPSPs evoked in the analyzed motoneurons

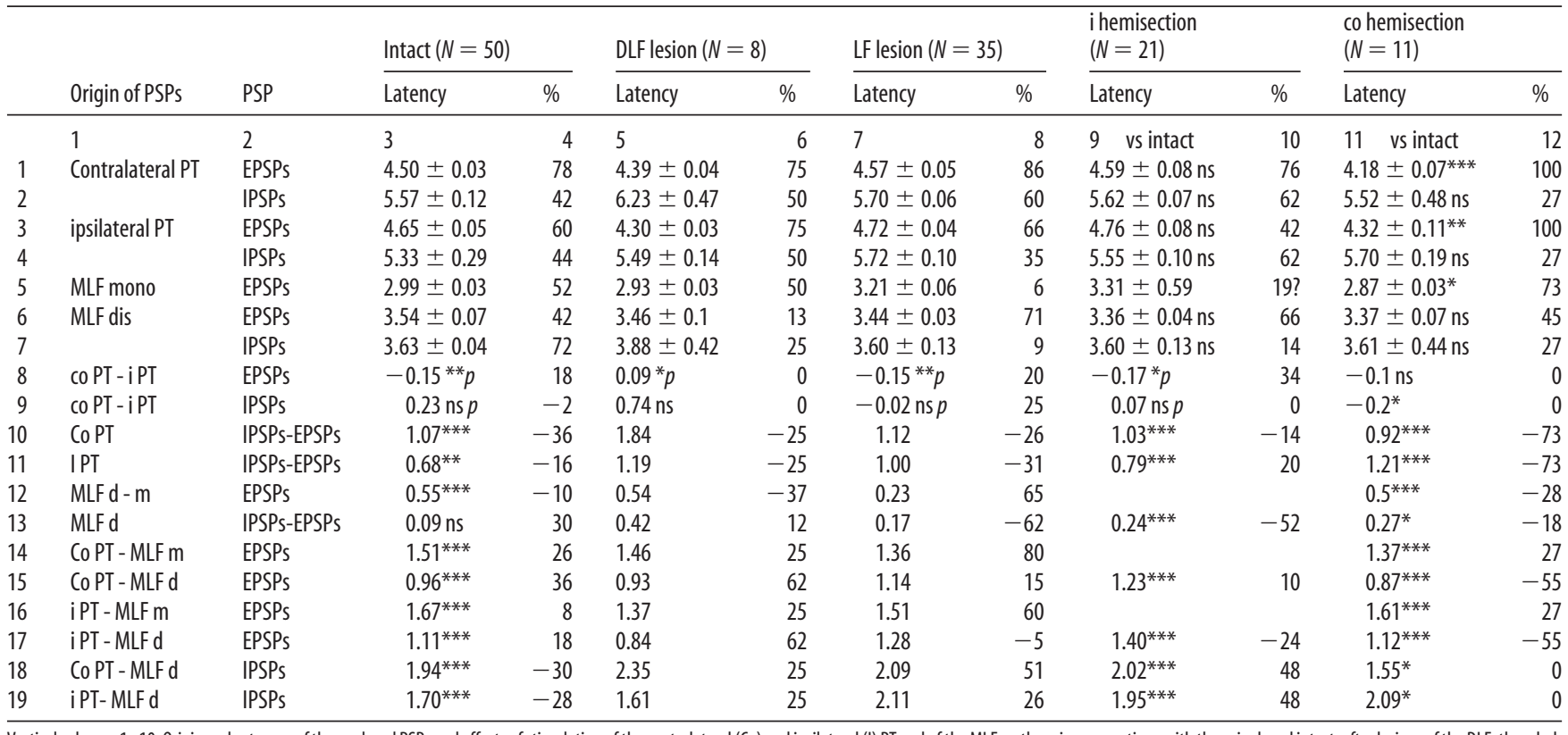

Vertical columns 1-10, Origin and category of the analyzed PSPs and effects of stimulation of the contralateral (Co) and ipsilateral (I) PT and of the MLF on them in preparations with the spinal cord intact, after lesions of the DLF, the whole lateral funiculus (LF) and both the lateral and ventral funiculi (hemisection). Horizontal rows 1-7, Latencies (means and SEM) and incidence of EPSPs and IPSPs evoked from the contralateral and ipsilateral PT and from the MLF. Horizontal rows $8-19$ in columns $3,5,7,9$, and 11 , Comparisons of latencies and incidence of EPSPs and IPSPs and of those evoked from the coPT, iPT, and MLF. Statistically significant differences are: ${ }^{*} p<0.05$ ), ${ }^{* *} p 0.001-0.01$ ), and ${ }^{* * *} p<0.001$ ); $\mathrm{ns}$, statistically not significant differences; $t$ test for paired two samples (when the number of pairs allowed such test) by $p$. Horizontal rows $8-19$ in columns $4,6,8,10$, and 12, Differences in incidence (\% of motoneurons) in the same combinations. 
connections between both the PT and reticulospinal (RS) neurons and spinal neurons were left intact ("intact preparations") and after various lesions of the spinal cord.

PSPs were attributed to either ipsilateral or contralateral PT fibers when they were evoked at intensities at which the collision tests illustrated in Figure 1 (see also Jankowska et al., 2006, their Fig. 2) did not reveal any spread of current from the pyramid on one side of the midline to the other. In the 7 experiments in which such tests were made, no spread of current was found at intensities up to $100 \mu \mathrm{A}$ (in 1 experiment), up to $110 \mu \mathrm{A}$ (in 5 experiments) and up to $150 \mu \mathrm{A}$ (in 1 experiment). The main reported effects are therefore based on effects of stimulation of the PTs at $100 \mu \mathrm{A}$ or less; stronger stimuli (150 or $200 \mu \mathrm{A})$ were used primarily to verify lack of PSPs of PT origin when they failed to be evoked at $100 \mu \mathrm{A}$.

In contrast to effects evoked from PTs, those evoked from the MLF could not be attributed to RS neurons descending either to the left or to right of the midline because of unavoidable spread of current from one side to another when the electrodes were placed $<0.6 \mathrm{~mm}$ from the midline (Gustafsson and Jankowska, 1976). The exceptions were effects of MLF stimuli applied after hemisection of the spinal cord when only fibers descending on one side were left intact.

Analysis. Both the original data and averages of 10-40 single records were stored on line. Peak amplitudes or the areas of the averaged potentials were measured off line using a software sampling and analysis system (designed by E. Eide, T. Holmström and N. Pihlgren, University of Göteborg). Differences between samples were assessed for statistical significance using Student's $t$ test (for two samples assuming equal variances). ${ }^{\star} p<0.05,{ }^{* *} p<0.01,{ }^{* *} p<0.001$. Mean values are given as means \pm SEM.

\section{Results}

The network of neurons that were investigated regarding their role in mediating actions of PT neurons on longissimus dorsi motoneurons and the location of the lesions performed to verify, or to exclude, their involvement are outlined in Figure 2. We will demonstrate that the most direct coupling between PT neurons and longissimus dorsi motoneurons is disynaptic or trisynaptic and hence as direct as the coupling between the PT neurons and proximal limb muscles. It will also be shown that effects of contralateral and ipsilateral PT neurons on these motoneurons are comparable, allowing back motoneurons to be controlled by both ipsilaterally and contralaterally located corticospinal neurons and that reticulospinal neurons with axons descending within the medial longitudinal fascicle (MLF) are the main relay neurons of $\mathrm{PT}$ actions on axial motoneurons.

PSPs evoked from PTs in preparations with intact spinal cord Short-latency PSPs from the contralateral PT

Stimuli applied within the contralateral PT evoked short-latency EPSPs and/or IPSPs in all but two of 50 motoneurons tested. EPSPs were recorded in $39(78 \%)$ motoneurons. Figure $3 \mathrm{H}-\mathrm{J}$ illustrates the main features of these EPSPs, in particular that EPSPs evoked by successive stimuli were distinct and showed marked temporal facilitation. In the illustrated motoneurons small EPSPs were evoked already by the second stimuli and appeared with increasing amplitudes and steeper rising phases after the third and fourth stimuli. However, Figure $4 A$ (black columns) shows that short-latency EPSPs from the contralateral PT were most often evoked only after the third or fourth PT stimuli, rarely appearing after the second stimulus and never after the first stimulus. These features, together with latencies of $4.16-4.99 \mathrm{~ms}$ from the third or fourth stimuli (mean latency $4.50 \pm 0.03 \mathrm{~ms}$, Table 1 column 3, row 1) characterize these EPSPs as being evoked either disynaptically or trisynaptically (see Discussion). They were evoked at stimulus intensities as low as
Ipsilateral PT $100 \mu \mathrm{A}$

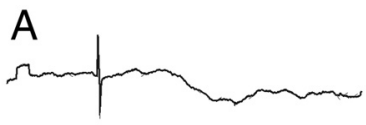

$7.4 \mathrm{~ms}$

B

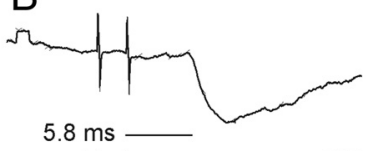

C
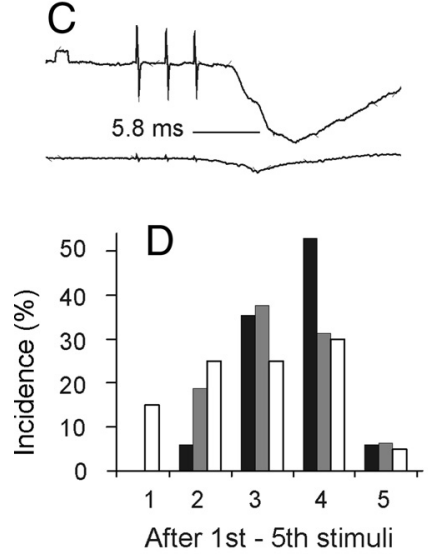

coPT ( $\square$ ), ipsi PT ( $\square)$ MLF ( $\square)$

\section{Contralateral PT $100 \mu \mathrm{A}$}
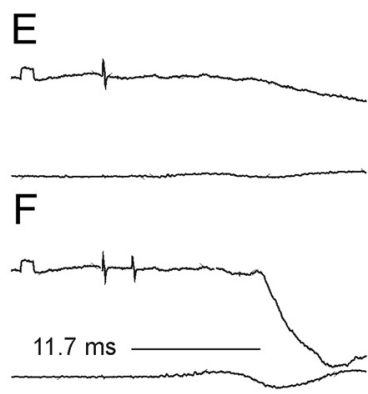

G

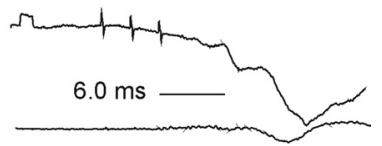

$\mathrm{H}$

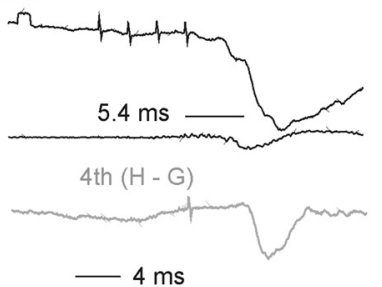

$-4 \mathrm{~ms}$
Figure 5. Examples of IPSPs evoked from the ipsilateral and contralateral PTs and their incidence. Upper and lower traces in $\boldsymbol{A} \boldsymbol{H}$ are intracellular records from a motoneuron and cord dorsum potentials, respectively, in a preparation with the spinal cord intact. Averages of 20 records. $\boldsymbol{A}-\boldsymbol{C}, \boldsymbol{E}-\boldsymbol{H}$, IPSPs evoked by increasing numbers of stimuli applied within the ipsilateral and contralateral PT. D, Proportions of motoneurons in which IPSPs with latencies not exceeding $6 \mathrm{~ms}$ appeared following successive stimuli $(100 \mu \mathrm{A})$ applied within the ipsilateral and contralateral PT and within the MLF when the previous stimuli were not effective in preparations with spinal cord intact, as in Figure $4 \mathrm{~A}$ for EPSPs. Other indications are as in Figure 3.

$40-60 \mu \mathrm{A}$, although longer trains of such weaker stimuli were required (Fig. 3, compare $J-L$ ).

Short-latency IPSPs followed stimulation of the contralateral PT in 9 motoneurons; they are illustrated in Figure 5. However, in 12 motoneurons the decay phases of disynaptic EPSPs evoked by the third or fourth stimuli were faster than of those evoked by the earlier stimuli, in particular when the motoneurons were depolarized by $10-20 \mathrm{nA}$. This was taken to indicate that IPSPs were evoked in parallel with these EPSPs but at slightly longer latencies and hence that IPSPs were evoked in a total of 21 of $50(42 \%)$ motoneurons. As shown in Table 1 (column 3, rows 2 and 10), latencies of IPSPs were $\sim 1$ ms longer than those of EPSPs $(5.50 \pm 0.12 \mathrm{~ms})$.

The incidence of short-latency IPSPs following successive stimuli in a train (Fig. 5D) resembled that of EPSPs. They appeared most often following the third or fourth, rarely after the second stimuli and were never evoked after single stimuli.

\section{Short-latency PSPs from the ipsilateral PT}

Short-latency EPSPs from the ipsilateral PT were recorded in a smaller proportion of motoneurons (30 of 50; 60\%) than from the contralateral PT. Similar to EPSPs evoked from the contralateral PT, they were most often evoked only by the third or fourth stimuli (Figs. $3 B-D, 4 A$, histogram). They were also temporally facilitated, showed similar time characteristics and amplitudes 


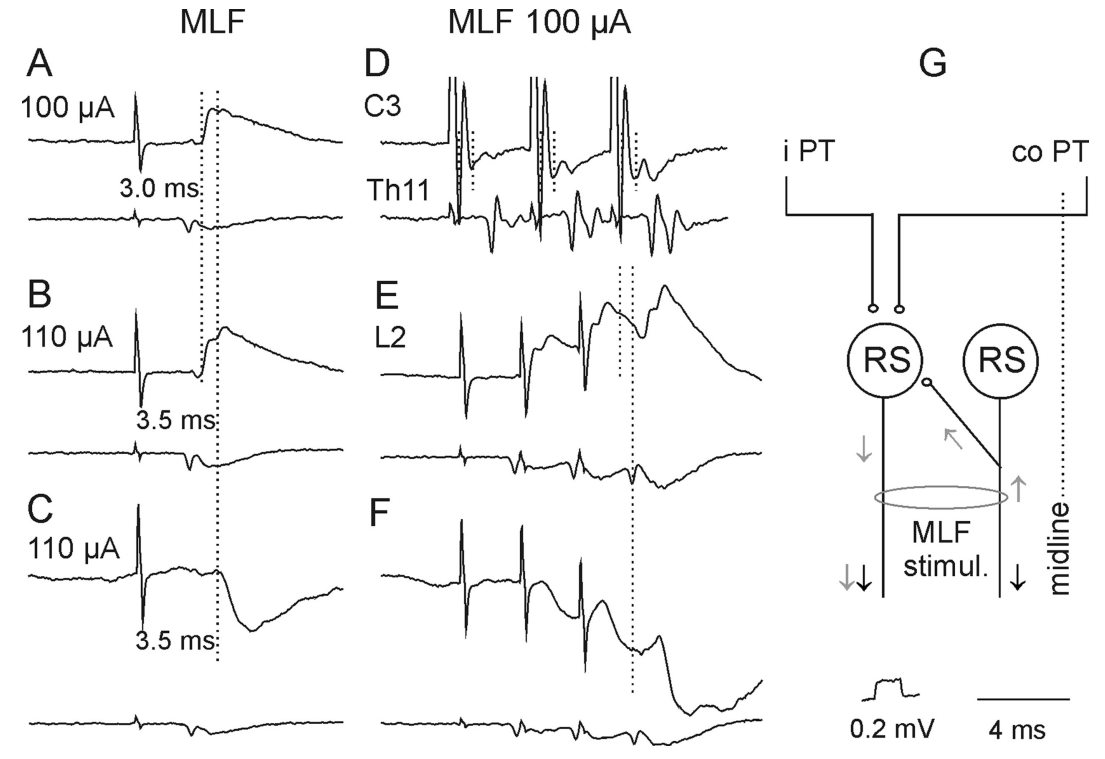

Figure 6. $\boldsymbol{A}-\boldsymbol{H}$, Examples of descending volleys and PSPs evoked from the MLF. $\boldsymbol{D}$ and lower traces in the remaining panels show descending volleys recorded at cervical, thoracic, and lumbar segmental levels, respectively. Note that the early volleys in $\boldsymbol{D}$ were followed by later volleys, indicated by two vertical dotted lines, and that the second volleys were larger after each successive stimulus. Upper traces in $\boldsymbol{A}, \boldsymbol{B}$, and $\boldsymbol{E}$ and in $\boldsymbol{C}$ and $\boldsymbol{F}$, Intracellular records from two motoneurons in the same preparation (with the spinal cord intact). Note that the early components of EPSPs (indicated by the first dotted line in $\boldsymbol{A}, \boldsymbol{B}$, and $\boldsymbol{E}$ ) were followed by later components (indicated by the second dotted lines). Note also similar latencies of the later components of the EPSPs and of the IPSPS with respect to the last stimuli. $\mathbf{G}$, Diagram of a neuronal network allowing direct actions of descending volleys (black arrows) to be followed by recurrent actions evoked via axon collaterals of fibers stimulated within the MLF on RS neurons (gray arrows) and activation of the same neurons by axon collaterals of PT fibers (modified from Edgley et al., 2004, their Fig. 1). Other indications are as in $\boldsymbol{F}$.

(Fig. $4 B$ ) and were evoked at similar latencies (Table 1, column 3, rows 3 and 10) and thresholds (Fig. 3D-F).

Short-latency IPSPs were observed in 22 of 50 (44\%) motoneurons. They were the sole effect in 7 motoneurons (with examples in Fig. 5 B, C) and followed EPSPs in an additional 15 motoneurons. The IPSPs were evoked at practically the same latencies as IPSPs from the contralateral PT (Table 1 column 3, rows 4 and 8 ) and were $\sim 0.7 \mathrm{~ms}$ longer than latencies of EPSPs (row 11).

Long-latency PSPs from the contralateral and ipsilateral PTs In addition to short-latency PSPs, longer-latency EPSPs and IPSPs often followed the second and sometimes the first stimuli. They are illustrated in Figure 3, $G$ and $H$, and in Figure $5 F$, respectively. Long-latency EPSPs (evoked at 9.73-15.8 ms) were more commonly observed following stimulation of the contralateral PT than of the ipsilateral PT, and were most conspicuous when the short-latency EPSPs or IPSPs were small or absent. However, the appearance of short-latency PSPs induced by the third-fifth stimuli made it difficult to distinguish or determine the presence of the longer-latency PSPs evoked by earlier stimuli.

\section{PSPs evoked from the MLF}

Short-latency EPSPs and/or IPSPs from the MLF were found in all motoneurons tested. In contrast to those evoked from PTs, EPSPs from the MLF were most frequently evoked by single stimuli (Fig. 4A) and at much shorter latencies. EPSPs in 23 of 44 motoneurons were evoked at latencies $0.5-0.8 \mathrm{~ms}$ from the descending MLF volleys and the earliest components were evoked by successive stimuli and displayed fairly constant amplitudes, indicating that they were evoked monosynaptically. Such EPSPs were recorded in 6 of 12 motoneurons tested when the stimuli were applied in the ipsilateral MLF and in 17 of 32 motoneurons from the contralateral MLF, but under conditions when ipsilaterally descending fibers could be coexcited (see Materials and Methods). Later components of 10 of these EPSPs, with examples in Figure $6 A, B$, and $E$, were delayed with respect to the onset of the earliest components by $0.4-0.9 \mathrm{~ms}$ (Table 1 , column 3, row 12) and appeared at latencies 1.0-1.4 $\mathrm{ms}$ from the descending volleys. EPSPs at the same latencies were also evoked in additional 11 motoneurons. In contrast to the earliest EPSPs, all the longer-latency EPSPs showed marked temporal facilitation and both these features indicated that they were evoked disynaptically.

IPSPs were evoked instead of EPSPs in 9 motoneurons. However in 25 motoneurons IPSPs were concluded to follow EPSPs, as judged from the faster decay phases of EPSPs evoked by the secondfourth than the first stimuli, thereby indicating the incidence of the IPSPs in up to 34 of $44(72 \%)$ of the motoneurons. The segmental latencies of the 9 IPSPs not preceded by EPSPs (1.0-1.7 ms) were on average $0.61 \mathrm{~ms}$ longer than the latencies of monosynaptic components of EPSPs (Table 1 , column 3, row 12) and only $0.09 \mathrm{~ms}$ longer than the latencies of EPSPs classified as evoked disynaptically (Table 1 column 3, row 13; Fig. 6C,F). Latencies of IPSPs overlapping with EPSPs generally fell within the same range as latencies of IPSPs that were not preceded by EPSPs.

IPSPs of MLF origin would have to be mediated by spinal premotor inhibitory interneurons as no evidence has been found for projections of inhibitory reticulospinal neurons to lumbar segments (Grillner et al., 1968; Peterson et al., 1979; Stecina and Jankowska, 2007; but see Baldissera and Roberts, 1975). In contrast, EPSPs evoked disynaptically from the MLF could be relayed via reticulospinal neurons activated by recurrent axon collaterals of fibers stimulated within the MLF, as indicated in Figure $6 G$, as well as by spinal premotor interneurons. Records illustrated in Figure 6 show the rationale behind considering both of these possibilities. The appearance at the $\mathrm{C} 3$ level of second components $\sim 1 \mathrm{~ms}$ after the earliest components of descending volleys following stimulation of the MLF (Fig. $6 D$, second dotted line) indicates that they reflected activation of more rostrally located neurons and would therefore be compatible with synaptic activation of additional RS neurons by the second and third stimuli. Although marginal after weak single stimuli, they increased with stronger stimuli and after successive stimuli. If the second components of EPSPs evoked by MLF stimuli (Fig. 6B, E, indicated by the second dotted lines) were evoked by the second components of the descending volleys, both should appear at a similar delay with respect to the earlier components, as was indeed the case. Consequently, both the earlier and the later components of MLF-evoked EPSPs could have been evoked by using direct connections between RS neurons and motoneurons indicated in the diagram of Figure 2. However, the possibility that these disynaptic EPSPs are 
evoked via spinal interneurons interposed between RS neurons and motoneurons cannot be excluded on the basis of their latencies, as these fell within the same range as latencies of disynaptically evoked IPSPs (Fig. 6E,F; Table 1 column 3, row 15) which had to be relayed by spinal interneurons.

\section{Which neurons relay $\mathrm{PT}$ actions?}

Three main categories of neurons might relay disynaptically evoked excitation or inhibition of longissimus lumborum motoneurons by PT neurons. In Figure 2 they are represented by green, gray and black relay neurons, respectively. Green neurons denote reticulospinal (RS) neurons, while gray and black neurons represent two categories of spinal relay neurons. To differentiate between PT actions mediated via these neurons we used three different approaches. The first was to compare PSPs evoked from the two PTs after lesions eliminating direct contributions of either RS neurons (by transection of the MLF), or of PT neurons (by transection of PT fibers at a spinal level) with those evoked in preparations in which the spinal cord remained intact. The second was to investigate the convergence of PT and MLF actions on neurons hypothesized to relay these actions at spinal and supraspinal levels, and the third to compare latencies of PSPs of MLF and of PT origin in these preparations.
Before MLF lesion
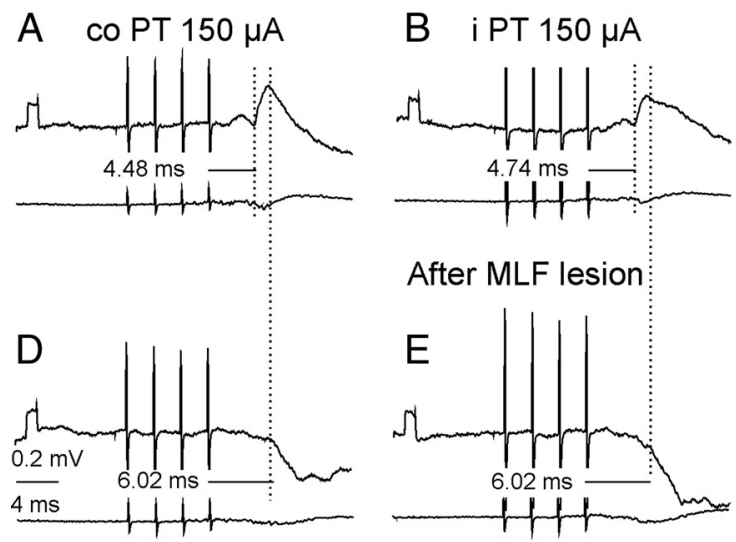

C MLF $150 \mu \mathrm{A}$

After MLF lesion:
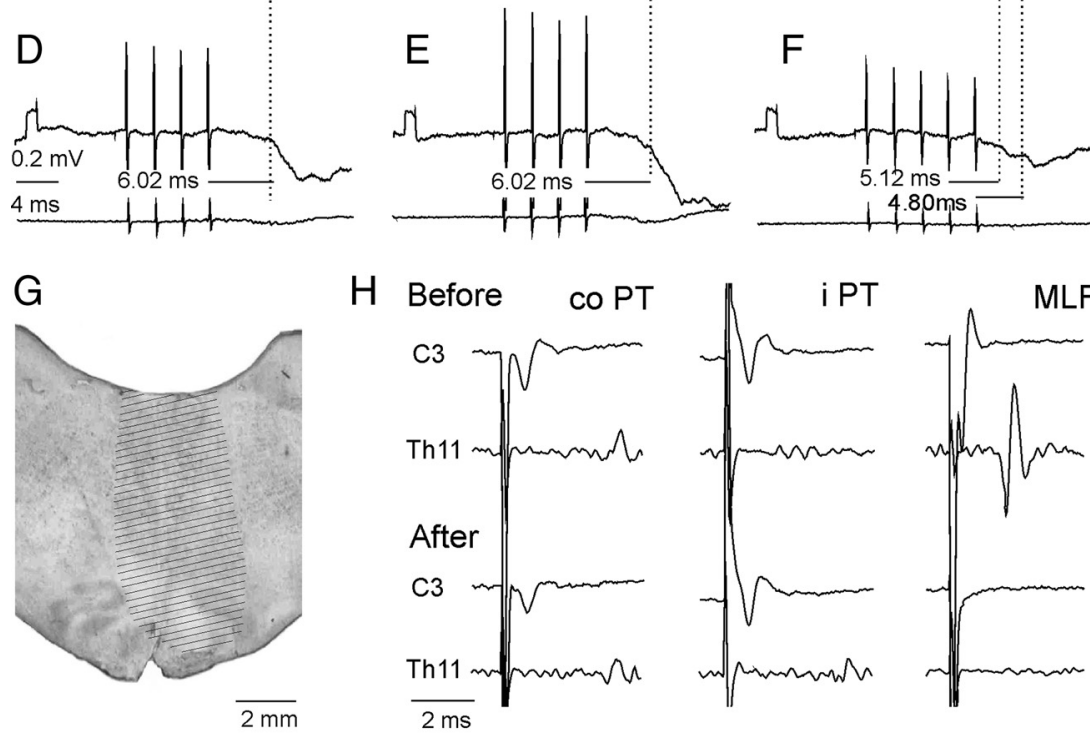

Figure 7. Effects of transection of the MLF on synaptic actions evoked by stimuli applied in the PTs and in the MLF. $\boldsymbol{A}-\boldsymbol{C}$ and $\boldsymbol{D}-\boldsymbol{F}$, Intracellular records from two motoneurons and records from the cord dorsum in the same experiment. They were obtained before and after transection of the MLF $\sim 5 \mathrm{~mm}$ caudal to the MLF stimulation site. $\boldsymbol{G}$, Maximal extent of the lesion. $\boldsymbol{H}$, Records of descending volleys from the left lateral funiculus before and after the lesion (extended until the volleys following MLF stimulation disappeared). Note that the lesion abolished EPSPs but not IPSPs evoked by contralateral (co) PT, ipsilateral (i), and MLF stimuli. Note also that it abolished early volleys from the MLF but did not abolish, or only partially abolished, cord dorsum potentials following PT stimuli.

\section{Effects of transection of the MLF}

After transection of the MLF, PT stimuli failed to evoke EPSPs in any of the 27 motoneurons tested. As illustrated in Figure 7, stimulation of either the contralateral or ipsilateral PT evoked only IPSPs. These were evoked by the third, fourth or fifth PT stimuli at latencies of $6.00 \pm 0.27 \mathrm{~ms}$ and $6.37 \pm 0.24$ from the contralateral and ipsilateral PT, respectively, and hence closer to the short-latency than the long-latency IPSPs described above. In the two experiments in which the MLF was lesioned, IPSPs were evoked in 7 of 16 and 10 of 11 motoneurons.

These results indicate that EPSPs evoked by PT stimuli are mediated predominantly, if not exclusively, via RS neurons. The same might be also true for the shortest latency IPSPs. The effects of MLF lesions could also, at least to some extent, be secondary to changes in the state of the animal. However there were no direct indications of this because the lesions did not result in changes in heart rate or blood pressure. In addition, control records from unidentified neurons located more dorsally revealed that the MLF lesions did not abolish all short-latency effects of PT neurons. In these neurons EPSPs and IPSPs were evoked by PT stimulation at latencies as short as those in motoneurons recorded in intact preparations.

Effects of transection of ipsilaterally descending PT fibers

Effects of elimination of ipsilaterally descending PT fibers by transection of the dorsolateral part of the lateral funiculus or of the whole lateral funiculus at a cervical or thoracic level (see
Materials and Methods) were strikingly minor, in particular as regards excitatory effects of PT stimulation. As shown in Table 1 (cf. column 4, 6 and 8, rows 1 and 3) the percentages of motoneurons in which EPSPs were evoked from either of the PTs after lesions to the lateral funiculus were similar to those of motoneurons recorded when the ipsilateral PT fibers were intact. Latencies of these potentials were likewise similar (any differences were not statistically significant) and amplitudes of EPSPs (Fig. 4D,F) were comparable to amplitudes of EPSPs recorded in preparations in which the spinal cord was intact (Fig. $4 B$ ) even though longer trains of stimuli were needed to evoke them after LF lesions (Fig. 4E). The only more conspicuous differences appeared to concern short-latency IPSPs evoked from the contralateral PT. Figure 8 illustrates the finding that weaker IPSPs were evoked from the contralateral PT than from the ipsilateral PT (Fig. 8, compare $K, L$ and $N, O$ ), until the length of the train of contralateral PT stimuli was increased (Fig. 8, compare $L$ and $M$ ).

To investigate a potential contribution of ipsilaterally descending propriospinal neurons located between the $\mathrm{C} 3$ and Th11 levels and of ipsilaterally and contralaterally located lumbar interneurons to PT actions, effects of lesions restricted to the dorsal part of the lateral funiculus at the C3 level or to the entire lateral funiculus at the Th11 level or including both the lateral and ventral funiculus (hemisection) at the Th11 level were also compared. 

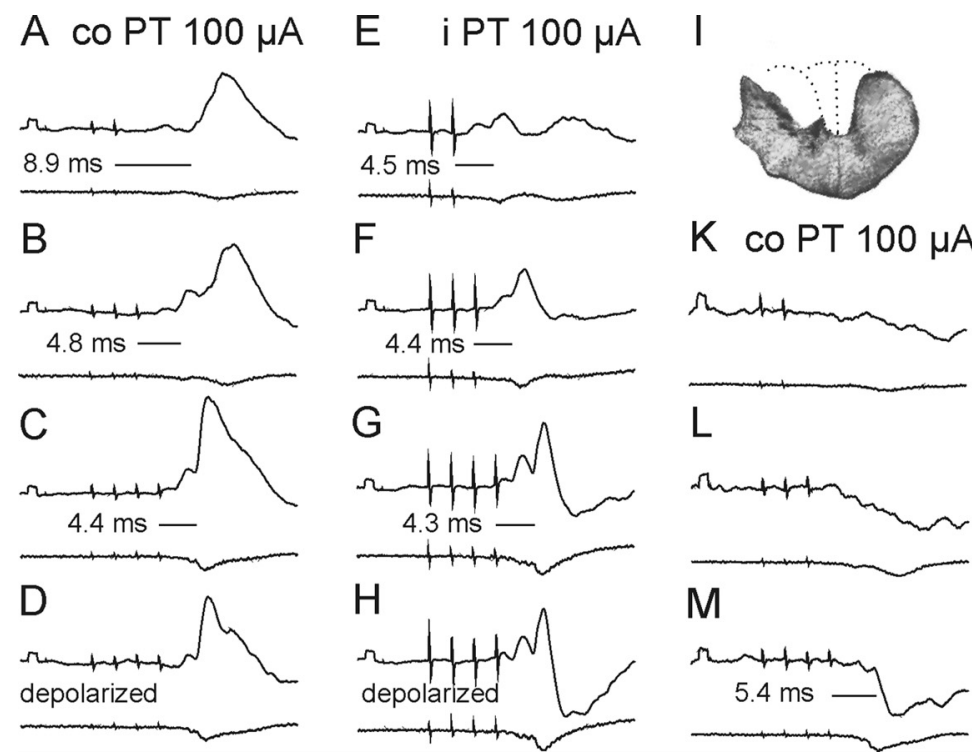

J co PT $100 \mu \mathrm{A}$

$\mathrm{F}$

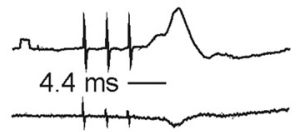

G
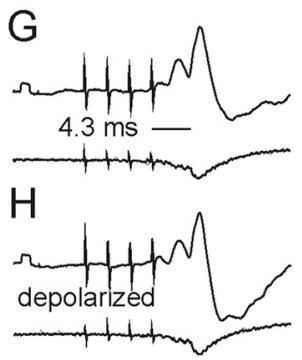
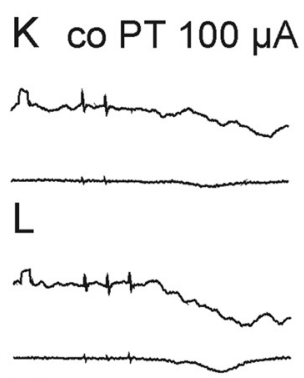

$\mathrm{M}$

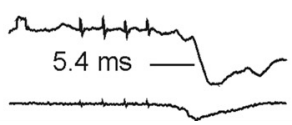

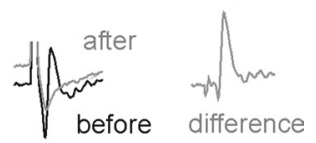

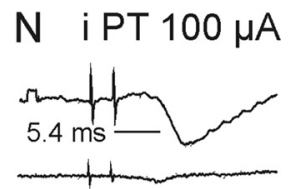

0

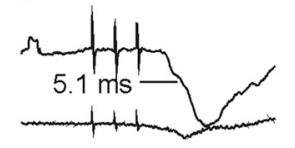

s. $0.2 \mathrm{mV}$

$-4 \mathrm{~ms}$

Figure 8. $\quad \boldsymbol{A}-\mathbf{0}$, PSPs evoked by PT stimulation after a lesion of the dorsal part of the lateral funiculus (DLF). In all panels, except $J$, upper traces are intracellular records from two motoneurons $(\boldsymbol{A}-\boldsymbol{H}$ and $\boldsymbol{K}-\mathbf{0}$, respectively) and lower traces are from the cord dorsum in the L2 segment, with the same format as in Figures 3 and 5 . All these records were obtained after a lesion of the dorsolateral part of the left lateral funiculus (DLF) in the rostral half of the C 3 segment. A reconstruction of this lesion is shown in I.J, Cord dorsum potentials recorded in the C 4 segment, evoked by stimuli applied in the co PT before (black) and after (gray) the lesion. The difference between these potentials is shown to the right. Note that EPSPs were evoked as efficiently from the co PT as from the ipsilateral (i) PT (and at the same latencies), but IPSPs required a longer train of stimuli (and were evoked at longer latencies, i.e., less efficiently).

Data in columns 3, 5 and 7 in Table 1 did not reveal any statistically significant differences that could indicate that ipsilaterally descending propriospinal neurons located in the C3-4 segments (Illert et al., 1978; Lundberg, 1979) were involved in relaying PT actions in addition to ipsilaterally descending reticulospinal tract fibers. The only more marked effect of lesions of the lateral funiculus was a reduced effectiveness of the first-third stimuli in the trains of PT stimuli, as most of the EPSPs appeared only following the fourth or even later stimuli (Fig. $4 E$ ), and smaller amplitudes of EPSPs from the ipsilateral than contralateral PT (statistically significant at $p<0.02$ ) (Fig. $4 F$ ). However, this could be attributed to effects secondary to the transection of reticulospinal tract fibers descending within the lateral funiculus, as an additional decrease in the efficiency of PT stimuli occurred when all ipsilaterally descending reticulospinal tract fibers were transected (Fig. 4G,H) and amplitudes of EPSPs evoked from the MLF as well as from the PTs were significantly reduced.

\section{Indications for common relay neurons of synaptic actions from the two PTs and the MLF}

Two main observations indicate that synaptic actions evoked from the PTs and from the MLF were mediated by the same neurons: the qualitatively similar synaptic actions evoked in individual motoneurons from these three sources and the mutual facilitation of these actions. Opposite effects from the ipsilateral PT and contralateral PT were only encountered in one motoneuron.

Examples of the similar timing of EPSPs or IPSPs evoked from the two PTs are shown in Figures 3, 5, and 8, while EPSPs or IPSPs evoked in parallel from the PTs and from the MLF are illustrated in Figure 7. The most striking similarities between synaptic actions from both PTs and from the MLF occurred in the case of 9 motoneurons in which IPSPs were evoked instead of EPSPs. However, such qualitative similarities did not exclude quantitative differences. As shown in Table 1 (rows 1 and 3), stimulation of the contralateral PT evoked EPSPs in a larger proportion of motoneurons than stimulation of the ipsilateral PT, even though mean amplitudes of these EPSPs (Figs. 3, 4B, D, F, H, 8, 9) were comparable. Differences were also found in the degree of inhibition evoked from the two PTs, but in this case with a tendency for IPSPs evoked from the ipsilateral PT to be larger, both when the spinal cord was intact (Figs. 3, 5) and after lesions of crossed PT fibers (Figs. 8, 9).

Joint application of subthreshold stimuli in the two PTs often rendered them effective, as has previously been demonstrated for effects of PT stimulation on hindlimb motoneurons (Jankowska et al., 2005). Both excitatory and inhibitory actions from the two PTs were facilitated in all 6 motoneurons tested, with examples in Figure $9 C-F$ (middle and right columns). Mutual facilitation also occurred between effects of subthreshold PT and MLF stimuli in all 4 motoneurons tested, with an example in Figure $9 C-F$ (left column), as previously found in hindlimb motoneurons (Edgley et al., 2004). Note that the facilitated PSPs (the onset indicated by dotted lines) appeared at the same latencies as EPSPs from the contralateral PT (A left) or ipsilateral PT (B middle) and as IPSPs from either the contralateral PT (C right) or ipsilateral PT (D right).

Such facilitatory effects provide evidence for converging input from the ipsilateral and contralateral PTs and from the PTs and the MLF on common relay neurons (Lundberg, 1964). However, it does not reveal where these common relay neurons are located. For example, disynaptic EPSPs appearing on joint stimulation of contralateral (co) PT and MLF (Fig. 9E in the left column) could have been relayed by premotor interneurons as well as by RS neurons coexcited by PT fibers and recurrent collaterals of MLF fibers (Fig. 6G). The latter possibility was in fact made particularly likely by the increase of the descending volleys illustrated in Figure 9E (arrow). However, coexcitation of individual spinal interneurons by contralateral and ipsilateral PT fibers and by reticulospinal fibers running in the MLF has previously been demonstrated (Jankowska et al., 2006; Jankowska and Stecina, 2007; Stecina et al., 2008a). 
Estimates of the relative contribution of ipsilaterally located interneurons and of commissural interneurons to PT actions were as difficult when the spinal cord remained intact as when it was hemisected. Theoretically, as shown the diagram in Figure 2, commissural interneurons would be likely to mediate crossed actions after an ipsilateral hemisection (Fig. 9, illustrated in the left column) and ipsilaterally located interneurons to mediate uncrossed actions after a contralateral hemisection (Fig. 9, illustrated in the middle and right columns) However, the possible synaptic actions of crossed axon collaterals of reticulospinal tract fibers (Matsuyama et al., 1999) (indicated by “?” in Fig. 2) make such interpretation less straightforward. Monosynaptic EPSPs evoked by contralaterally descending reticulospinal tract fibers after ipsilateral hemisection and thus attributable to such crossed collaterals were previously found in only $9 \%$ of hindlimb motoneurons (Jankowska et al., 2003) but they might have been evoked in as many as $19 \%$ of the present sample of motoneurons (Table 1 column 10, row 5), suggesting that they are more widespread in the rostral than in the caudal lumbar segments. Identifying these EPSPs as evoked monosynaptically was not unequivocal as their latencies (0.96-1.02 $\mathrm{ms}$ ) fell at the border between latencies of monosynaptic and disynaptic EPSPs and they were tightly followed by later components that showed strong temporal facilitation. Nevertheless, if these EPSPs were evoked monosynaptically it would suggest that reticulospinal tract fibers not only affect motoneurons but also interneurons and via these, contribute to disynaptic EPSPs and IPSPs evoked in back motoneurons.

\section{Discussion}

The results of this study reveal (1) potent monosynaptic and disynaptic coupling between reticulospinal neurons and motoneurons innervating erector spinae muscles and (2) di-, tri- and polysynaptic coupling between both contralateral and ipsilateral pyramidal tract neurons and these motoneurons. The results further provide evidence that pyramidal actions on erector spinae motoneurons may be relayed by both RS neurons and spinal interneurons, similar to their actions on hindlimb motoneurons (Lundberg, 1979; Edgley et al., 2004; Jankowska et al., 2006).

\section{Coupling between corticospinal and reticulospinal neurons and erector spinae motoneurons}

As shown in the Results section, stimulation of reticulospinal fibers in the MLF-evoked monosynaptic EPSPs in the majority of motoneurons when ipsilaterally descending pathways were intact. However, EPSPs were also evoked at only marginally longer latencies when these pathways were transected at the Th11 level of the spinal cord. Their presence would thus indicate a nonnegligible contribution of crossed collaterals of reticulospinal neurons (Matsuyama et al., 1999) to effects relayed via reticulospinal tract fibers. Disynaptically evoked EPSPs of MLF origin could thus be mediated by premotor interneurons located on either side of the spinal cord. However, disynaptic EPSPs could also be the result of reexcitation of RS neurons contacting motoneurons by axon collaterals of fibers stimulated within the MLF (Edgley et al., 2004).

EPSPs following PT stimuli were evoked at both relatively short and longer latencies. The latencies of the earliest EPSPs were close to latencies of disynaptic EPSPs evoked in hindlimb motoneurons (4.70-4.81 ms) (Jankowska and Stecina, 2007; Stecina and Jankowska, 2007) but considerably shorter than latencies of trisynaptic EPSPs evoked via contralaterally descending pathways and commissural interneurons (6.07-6.72 ms) (Edgley et al., 2004; Jankowska et al., 2005). We may hence conclude that the shortest excitatory pathways between the PT neu- 
rons and back motoneurons are disynaptic. Latencies of IPSPs were consistently $\sim 1 \mathrm{~ms}$ longer, indicating that the earliest IPSPs were evoked via trisynaptic pathways, as would also be needed for the longer-latency components of EPSPs. The results are thus fully consistent with the hypothesis that they are mediated via reticulospinal neurons (Fig. 2).

The most conclusive argument in favor of RS neurons mediating PT actions on longissimus lumborum motoneurons is the disappearance of disynaptic EPSPs after a lesion of the MLF and the persistence of these actions following lesions of the PT fibers at a spinal level. As RS neurons are coactivated by PT neurons from both hemispheres ( $\mathrm{He}$ and $\mathrm{Wu}, 1985$; Matsuyama and Drew, 1997; Kably and Drew, 1998), and descend ipsilaterally as well as contralaterally (Mitani et al., 1988), activation by PT neurons from one hemisphere could result in excitation of back motoneurons on both sides of the spinal cord. Furthermore, as activity of individual RS neurons is associated with activation of muscles of both ipsilateral and contralateral arm and shoulder muscles (Schepens and Drew, 2006; Davidson et al., 2007) they might also exert such bilateral actions on back muscles.

In individual motoneurons of our sample, EPSPs, IPSPs, or EPSPs followed by IPSPS were most often evoked in parallel by stimulation of the PTs and of the MLF, as expected for actions mediated by RS neurons. The only observations that might be considered at variance with this were the observations of only marginally longer latencies of PSPs evoked by PT stimulation after ipsilateral hemisection of the spinal cord, i.e., when axons of the ipsilaterally descending RS neurons that directly connect with motoneurones were transected and only contralaterally descending pathways remained intact. However, two possible explanations of these EPSPs might make them compatible with being mediated by RS neurons. One would be that disynaptic PT actions evoked after ipsilateral hemisection are mediated by crossed collaterals of RS axons descending contralaterally, provided that the number of such collaterals is much greater in the rostral than in the caudal lumbar segments (see above). Another possibility would be that these PSPs were relayed tri- rather than disynaptically, via very fast conducting RS neurons descending contralaterally and via exceptionally effectively activated and fast conducting commissural interneurons.

As the appearance of both short- and long-latency EPSPs of $\mathrm{PT}$ origin was prevented by transection of axons of RS neurones descending within the MLF these results are in contrast to effects of MLF lesions on synaptic actions evoked by PT stimulation on hindlimb motoneurons (Stecina et al., 2008b) in which disynaptic EPSPs appeared both when the MLF was intact and after MLF transection, indicating that PT excitatory actions on hindlimb motoneurons are relayed in parallel by spinal interneurons and by RS neurons. The results of the present study do not, however, allow the exclusion of such parallel actions on back motoneurones, because weak actions of PT fibers on their target interneurons might be subthreshold, unless combined with additional input from RS neurons, and coexcitation by PT and MLF fibers has been demonstrated for other premotor interneurons (Jankowska and Stecina, 2007).

The situation was different for IPSPs evoked by PT stimulation after MLF lesions, indicating that they might be mediated by spinal interneurons. Nevertheless, these IPSPs could also be mediated by RS neurons with axons joining the MLF within the most caudal part of the medulla below the lesion, or by neurons projecting via the lateral reticulospinal tract (Petras, 1967; Peterson et al., 1979). The latter were reported to affect thoracic back motoneurons (Peterson et al., 1979) and actions of these neurons might extend to interneurons inhibiting lumbar back motoneurons.

Pathways between RS neurons and motoneurons via which IPSPs are evoked should include an additional inhibitory interneuron in view of lack of evidence that inhibitory RS neurons project as far caudally as the lumbosacral segments (Wilson and Yoshida, 1968; Peterson et al., 1979). To account for IPSPs relayed by RS neurons the most direct inhibitory pathways from RS neurons should thus be disynaptic. The interposed inhibitory interneurons could include interneurons located either ipsilaterally or contralaterally, including the lamina VII commissural interneurons with a previously demonstrated strong input from the MLF (Bannatyne et al., 2003) and bilaterally projecting inhibitory dorsal horn interneurons (Bannatyne et al., 2006, 2009). However, inhibitory PT actions appeared to depend on reticulospinal neurons to a lesser extent than excitatory actions as they were present after MLF lesions and were somewhat more affected by lesions of the ipsilaterally descending PT fibers. A contribution of spinal interneurons activated more directly by PT fibers would thus be more likely for inhibitory than for excitatory interneurons.

Together, the reported observations provide evidence that disynaptic EPSPs of PT origin in $\mathrm{m}$. longissimus lumborum motoneurons are mediated by RS neurons (although without excluding that they could be mediated in parallel by spinal interneurons) and leave open the possibility that trisynaptic EPSPs and IPSPs are mediated by both RS neurons and spinal interneurons.

\section{Comparison with data on humans}

Data from studies in humans using transcranial magnetic stimulation (TMS) to evoke contralateral and ipsilateral EMG responses in the erector spinae are broadly consistent with the observations of this study, as bilateral responses have frequently been observed, although not in all healthy subjects (Ferbert et al., 1992; Taniguchi and Tani, 1999; Fujiwara et al., 2001; Kuppuswamy et al., 2008). Support for ipsilateral control of the erector spinae comes also from observations in patients following stroke. For instance, in the majority of stroke patients investigated by Fujiwara et al. (2001), stimulation over the nonaffected hemisphere evoked bilateral responses, whereas TMS of the affected hemisphere failed to evoke responses in either ipsilateral or contralateral erector spinae.

The activation or unmasking of ipsilateral pathways after unilateral damage to the corticospinal tract, as occurs after stroke, may explain the lack of impairment of erector spinae functions after stroke (Horak et al., 1984; Dickstein et al., 2004). Activation of erector spinae in advance of the moving limb could also be related to preferential activation of proximal rather than distal muscles during postural adjustments (Marsden et al., 1999) and the less severe paresis in proximal than in distal muscles. Sparing of uncrossed pathways after stroke may thus play a role in recovery of trunk muscle function and, by stabilizing the trunk and its movements, assist in the recovery of limb muscle activity, during rehabilitation programs.

The descending control of back muscles may also become of increased importance when reflex control of these muscles is impaired in idiopathic low back pain. Both acute and chronic back pain is associated with atrophy of the deep back muscles and deficits in postural control (Hodges et al., 2009). Patients with chronic low back pain have in particular been reported to have poor postural control in the absence of visual feedback (Radebold 
et al., 2001). It might thus be useful if these deficits were at least partly compensated by a more effective control of back muscles by PT and RS neurons activated in conjunction with planned voluntary movements.

\section{References}

Alstermark B, Pinter MJ, Sasaki S (1985) Pyramidal effects in dorsal neck motoneurones of the cat. J Physiol 363:287-302.

Baldissera F, Roberts WJ (1975) Effects on the ventral spinocerebellar tract neurones from deiters' nucleus and the medial longitudinal fascicle in the cat. Acta Physiol Scand 93:228-249.

Bannatyne BA, Edgley SA, Hammar I, Jankowska E, Maxwell DJ (2003) Networks of inhibitory and excitatory commissural interneurons mediating crossed reticulospinal actions. Eur J Neurosci 18:2273-2284.

Bannatyne BA, Edgley SA, Hammar I, Jankowska E, Maxwell DJ (2006) Differential projections of excitatory and inhibitory dorsal horn interneurons relaying information from group II muscle afferents in the cat spinal cord. J Neurosci 26:2871-2880.

Bannatyne BA, Liu TT, Hammar I, Stecina K, Jankowska E, Maxwell DJ (2009) Excitatory and inhibitory intermediate zone interneurons in pathways from feline group I and II afferents: differences in axonal projections and input. J Physiol 587:379-399.

Brink EE, Jinnai K, Hirai N, Wilson VJ (1981) Cervical input to vestibulocollic neurons. Brain Res 217:13-21.

Caronni A, Cavallari P (2009a) Anticipatory postural adjustments stabilise the whole upper-limb prior to a gentle index finger tap. Exp Brain Res 194:59-66.

Caronni A, Cavallari P (2009b) Supra-spinal circuits shape inhibitory postural adjustments anticipating voluntary index-finger flexion. Exp Brain Res 198:19-28.

Chabran E, Maton B, Ribreau C, Fourment A (2001) Electromyographic and biomechanical characteristics of segmental postural adjustments associated with voluntary wrist movements. Influence of an elbow support. Exp Brain Res 141:133-145.

Cottingham SL, Femano PA, Pfaff DW (1988) Vestibulospinal and reticulospinal interactions in the activation of back muscle EMG in the rat. Exp Brain Res 73:198-208.

Davidson AG, Schieber MH, Buford JA (2007) Bilateral spike-triggered average effects in arm and shoulder muscles from the monkey pontomedullary reticular formation. J Neurosci 27:8053-8058.

Davies P (1990) Right in the middle-selective trunk activity in the treatment of adult hemiplegia. Berlin: Springer.

Deliagina TG, Orlovsky GN, Zelenin PV, Beloozerova IN (2006) Neural bases of postural control. Physiology (Bethesda) 21:216-225.

Deliagina TG, Beloozerova IN, Zelenin PV, Orlovsky GN (2008) Spinal and supraspinal postural networks. Brain Res Rev 57:212-221.

Dickstein R, Heffes Y, Laufer Y, Ben-Haim Z (1999) Activation of selected trunk muscles during symmetric functional activities in poststroke hemiparetic and hemiplegic patients. J Neurol Neurosurg Psychiatry 66:218-221.

Dickstein R, Shefi S, Marcovitz E, Villa Y (2004) Electromyographic activity of voluntarily activated trunk flexor and extensor muscles in post-stroke hemiplegic patients. Clin Neurophysiol 115:790-796.

Dum RP, Strick PL (1996) Spinal cord terminations of the medial wall motor areas in macaque monkeys. J Neurosci 16:6513-6525.

Edgley SA, Jankowska E, Hammar I (2004) Ipsilateral actions of feline corticospinal tract neurons on limb motoneurons. J Neurosci 24:7804-7813.

Ferbert A, Priori A, Rothwell JC, Day BL, Colebatch JG, Marsden CD (1992) Interhemispheric inhibition of the human motor cortex. J Physiol 453:525-546

Fujiwara T, Sonoda S, Okajima Y (2001) The relationships between trunk function and the findings of transcranial magnetic stimulation among patients with stroke. J Rehab Med 33:249-255.

Grillner S, Hongo T, Lund S (1968) Reciprocal effects between two descending bulbospinal systems with monosynaptic connections to spinal motoneurones. Brain Res 10:477-480.

Gustafsson B, Jankowska E (1976) Direct and indirect activation of nerve cells by electrical pulses applied extracellularly. J Physiol 258:33-61.

He XW, Wu CP (1985) Connections between pericruciate cortex and the medullary reticulospinal neurons in cat: an electrophysiological study. Exp Brain Res 61:109-116.

Hodges PW, Galea MP, Holm S, Holm AK (2009) Corticomotor excitability of back muscles is affected by intervertebral disc lesion in pigs. Eur J Neurosci 29:1490-1500.

Hongo T, Kudo N, Tanaka R (1975) The vestibulospinal tract: crossed and uncrossed effects on hindlimb motoneurones in the cat. Exp Brain Res 24:37-55

Horak FB, Esselman P, Anderson ME, Lynch MK (1984) The effects of movement velocity, mass displaced, and task certainty on associated postural adjustments made by normal and hemiplegic individuals. J Neurol Neurosurg Psychiatry 47:1020-1028.

Illert M, Lundberg A, Padel Y, Tanaka R (1978) Integration in descending motor pathways controlling the forelimb in the cat. 5. Properties of and monosynaptic excitatory convergence on $\mathrm{C} 3-\mathrm{C} 4$ propriospinal neurones. Exp Brain Res 33:101-130.

Jankowska E, Edgley SA (2006) How can corticospinal tract neurons contribute to ipsilateral movements? A question with implications for recovery of motor functions. Neuroscientist 12:67-79.

Jankowska E, Stecina K (2007) Uncrossed actions of feline corticospinal tract neurones on lumbar interneurones evoked via ipsilaterally descending pathways. J Physiol 580:133-147.

Jankowska E, Hammar I, Slawinska U, Maleszak K, Edgley SA (2003) Neuronal basis of crossed actions from the reticular formation upon feline hindlimb motoneurons. J Neurosci 23:1867-1878.

Jankowska E, Cabaj A, Pettersson LG (2005) How to enhance ipsilateral actions of pyramidal tract neurons. J Neurosci 25:7401-7405.

Jankowska E, Stecina K, Cabaj A, Pettersson LG, Edgley SA (2006) Neuronal relays in double crossed pathways between feline motor cortex and ipsilateral hindlimb motoneurones. J Physiol 575 527-541.

Kably B, Drew T (1998) Corticoreticular pathways in the cat. I. Projection patterns and collaterization. J Neurophysiol 80:389-405.

Kuppuswamy A, Catley M, King NK, Strutton PH, Davey NJ, Ellaway PH (2008) Cortical control of erector spinae muscles during arm abduction in humans. Gait Posture 27:478-484.

Lawrence DG, Kuypers HG (1968) The functional organization of the motor system in the monkey. I. The effects of bilateral pyramidal lesions. Brain 91:1-14.

Luccarini P, Gahery Y, Pompeiano O (1990) Cholinoceptive pontine reticular structures modify the postural adjustments during the limb movements induced by cortical stimulation. Arch Ital Biol 128:19-45.

Lundberg A (1964) Supraspinal control of transmission in reflex paths to motoneurones and primary afferents. Prog Brain Res 197-221.

Lundberg A (1979) Integration in propiospinal motor centre controlling the forelimb in the cat. In: Integration in the nervous system (Asanuma $\mathrm{H}$, Wilson VS, eds), pp 47-65. Tokyo: Igaru-Shoin.

Marsden JF, Farmer SF, Halliday DM, Rosenberg JR, Brown P (1999) The unilateral and bilateral control of motor unit pairs in the first dorsal interosseous and paraspinal muscles in man. J Physiol 521:553-564.

Massion J (1992) Movement, posture and equilibrium: interaction and coordination. Prog Neurobiol 38:35-56.

Massion J (1994) Postural control system. Curr Opin Neurobiol 4:877-887.

Matsuyama K, Drew T (1997) Organization of the projections from the pericruciate cortex to the pontomedullary brainstem of the cat: a study using the anterograde tracer Phaseolus vulgaris-leucoagglutinin. J Comp Neurol 389:617-641.

Matsuyama K, Mori F, Kuze B, Mori S (1999) Morphology of single pontine reticulospinal axons in the lumbar enlargement of the cat: a study using the anterograde tracer PHA-L. J Comp Neurol 410:413-430.

Mitani A, Ito K, Mitani Y, McCarley RW (1988) Morphological and electrophysiological identification of gigantocellular tegmental field neurons with descending projections in the cat: II. Bulb. J Comp Neurol 274:371-386.

Nyberg-Hansen R, Mascitti TA (1964) Sites and mode of termination of fibers of the vestibulospinal tract in the cat. J Comp Neurol 122:369-383.

Peterson BW, Pitts NG, Fukushima K (1979) Reticulospinal connections with limb and axial motoneurons. Exp Brain Res 36:1-20.

Petras JM (1967) Cortical, tectal and tegmental fiber connections in the spinal cord of the cat. Brain Res 6:275-324.

Radebold A, Cholewicki J, Polzhofer GK, Greene HS (2001) Impaired postural control of the lumbar spine is associated with delayed muscle response times in patients with chronic idiopathic low back pain. Spine (Phila Pa 1976) 26:724-730.

Schepens B, Drew T (2004) Independent and convergent signals from the 
pontomedullary reticular formation contribute to the control of posture and movement during reaching in the cat. J Neurophysiol 92:2217-2238.

Schepens B, Drew T (2006) Descending signals from the pontomedullary reticular formation are bilateral, asymmetric, and gated during reaching movements in the cat. J Neurophysiol 96:2229-2252.

Schepens B, Stapley P, Drew T (2008) Neurons in the pontomedullary reticular formation signal posture and movement both as an integrated behavior and independently. J Neurophysiol 100:2235-2253.

Stecina K, Jankowska E (2007) Uncrossed actions of feline corticospinal tract neurones on hindlimb motoneurones evoked via ipsilaterally descending pathways. J Physiol 580:119-132.

Stecina K, Jankowska E, Cabaj A, Pettersson LG, Bannatyne BA, Maxwell DJ (2008a) Premotor interneurones contributing to actions of feline pyramidal tract neurones on ipsilateral hindlimb motoneurones. J Physiol 586:557-574.
Stecina K, Slawinska U, Jankowska E (2008b) Ipsilateral actions from the feline red nucleus on hindlimb motoneurones. J Physiol 586:58655884 .

Taniguchi S, Tani T (1999) Motor-evoked potentials elicited from human erector spinae muscles by transcranial magnetic stimulation. Spine 24:154-156.

van Nes IJ, Nienhuis B, Latour H, Geurts AC (2008) Posturographic assessment of sitting balance recovery in the subacute phase of stroke. Gait Posture 28:507-512.

Wilson VJ, Yoshida M (1968) Vestibulospinal and reticulospinal effects on hindlimb, forelimb, and neck alpha motoneurons of the cat. Proc Natl Acad Sci U S A 60:836-840.

Zedka M, Prochazka A, Knight B, Gillard D, Gauthier M (1999) Voluntary and reflex control of human back muscles during induced pain. J Physiol 520:591-604. 